\title{
OPEN Improved magnetostriction in Galfenol alloys by aligning crystal growth direction along easy magnetization axis
}

\author{
Chao Zhou ${ }^{1}$, Yapeng Liu ${ }^{1}$, Kaiyun Chen ${ }^{1}$, Zhiyong Dai ${ }^{1}$, Tianyu Ma ${ }^{2}$, Yu Wang ${ }^{1}$, Shuai Ren ${ }^{1}$, \\ Junkai Deng ${ }^{1 \bowtie}$, Rui Zhang ${ }^{1}$, Fanghua Tian ${ }^{1}$, Yin Zhang ${ }^{1}$, Hao Zeng ${ }^{3}$ \& Sen Yang ${ }^{1 \bowtie}$
}

Galfenol (Iron-gallium) alloys have attracted significant attention as the promising magnetostrictive materials. However, the as-cast Galfenols exhibit the magnetostriction within the range of 20-60 ppm, far below the requirements of high-resolution functional devices. Here, based on the geometric crystallographic relationship, we propose to utilize the $90^{\circ}$-domain switching to improve the magnetostriction of Galfenols by tuning the crystal growth direction (CGD) along the easy magnetization axis (EMA). Our first-principles calculations demonstrate that Pt doping can tune the CGD of Galfenol from [110] to [100], conforming to the EMA. Then, it is experimentally verified in the $\left(\mathrm{Fe}_{0.83} \mathrm{Ga}_{0.17}\right)_{100-x} \mathrm{Pt}_{x}(x=0,0.2,0.4,0.6,0.8$ and 1.0$)$ alloys and the magnetostriction is greatly improved from $39 \mathrm{ppm}$ ( $x=0$, as-cast) to $103 \mathrm{ppm}(x=0.8$, as-cast) and $188 \mathrm{ppm}(x=0.8$, directionally solidified), accompanying with the increasing CGD alignment along [100]. The present study provides a novel approach to design and develop high-performance magnetostrictive materials.

The magnetostrictive effect, which realizes the conversion between mechanical energy and magnetic energy, provides the fundamental physics for important functional devices, such as sensors (e.g., stress/force/position sensors), actuators (e.g., linear motors) and transducers (e.g., energy harvesting devices) ${ }^{1,2}$.

Among the promising magnetostrictive materials, Fe-Ga (Galfenol) alloys have garnered significant research attention due to the excellent magnetoelastic properties and desirable comprehensive performance for mechanical working, such as excellent ductility and high mechanical strength ${ }^{3-7}$. Much effort has been dedicated to improving the magnetostriction of FeGa alloys, including enhancing the local magneto-crystalline anisotropy from the introduction of the tetragonal nanoheterogeneities (m-D03 phase $)^{8-14}$, application of prestress and magnetic annealing ${ }^{3,5,6,15-17}$, etc. But these methods result in design complexities and high fabrication cost.

The magnetostrictive effect originates from the lattice distortion caused by magnetoelastic coupling; such lattice distortion gives rise to the distorted magnetic domain along the easy magnetization axis (EMA). The magnitude of measured magnetostriction (only Joule magnetostriction is considered here ${ }^{18}$ ) is mainly determined from the $90^{\circ}$-domain switching ${ }^{19}$, and only the change of domain distortion component along the external field, which is usually parallel to the crystal growth direction (CGD), contributes to the measured magnetostriction during the switching of $90^{\circ}$-domain, as schematically illustrated in Fig. 1. Therefore, the CGD conforming to EMA (parallel to domain distortion) and the CGD deviating from the EMA, deliver different magnetostrictions.

In this work, we propose to utilize the $90^{\circ}$-domain switching to improve the magnetostriction of Galfenols by tuning the CGD along the easy magnetization axis (EMA). We shall show in order the $90^{\circ}$-domain-switching based magnetostrictive mechanism in ferromagnetic materials, the density functional theory (DFT) calculations to predict the CGD tuning by elemental doping, and the improved magnetostriction in FeGa alloys. Based on the results of the DFT calculations, we have synthesized Pt-doped FeGa alloys- $\left(\mathrm{Fe}_{0.83} \mathrm{Ga}_{0.17}\right)_{100-x} \mathrm{Pt}_{x}(x=0,0.2$, $0.4,0.6,0.8$ and 1.0) and demonstrated the enhancement effect in magnetostriction with the CGD aligning along EMA. The proposed methodology provides a general pathway to design high-performance magnetostrictive materials.

\footnotetext{
${ }^{1}$ School of Physics, MOE Key Laboratory for Nonequilibrium Synthesis and Modulation of Condensed Matter, State Key Laboratory for Mechanical Behavior of Materials, Xi'an Jiaotong University, Xi'an 710049, China. ${ }^{2}$ Frontier Institute of Science and Technology, Xi'an Jiaotong University, Xi'an 710049, China. ${ }^{3}$ Department of Physics, University at Buffalo, State University of New York, Buffalo, NY 14260, USA. ${ }^{\square}$ email: junkai.deng@xjtu.edu.cn; yang.sen@xjtu.edu.cn
} 

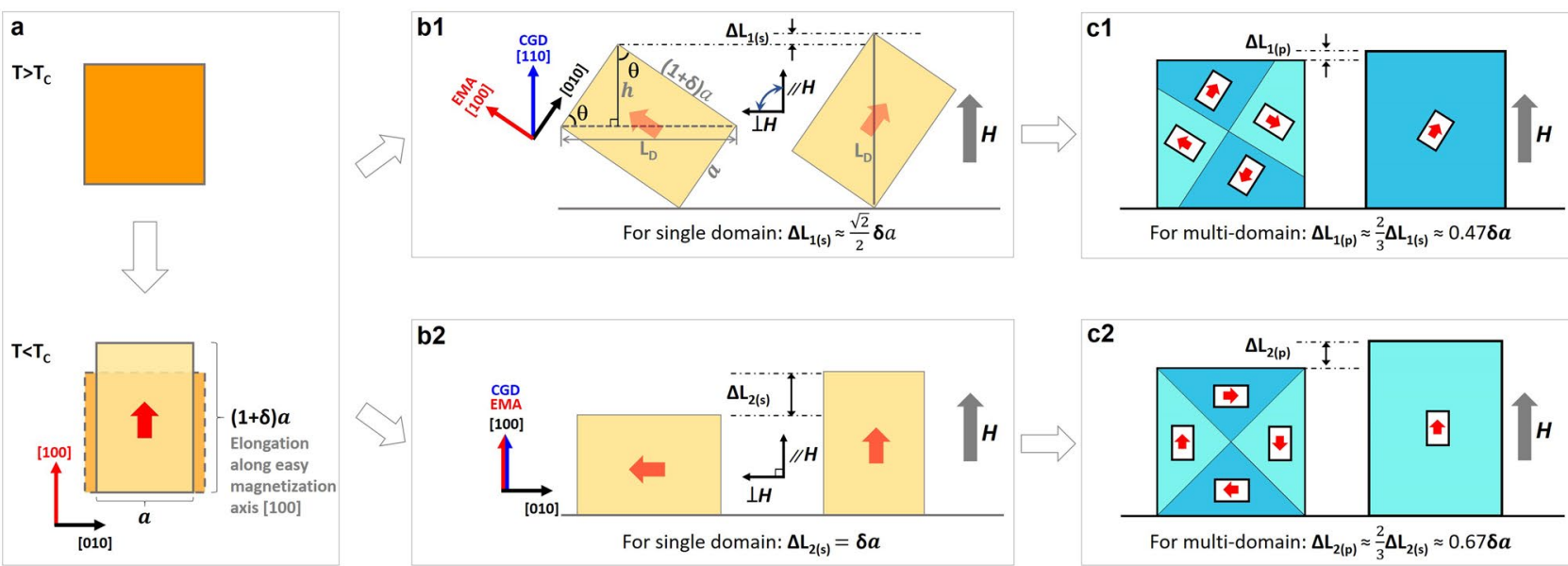

b2
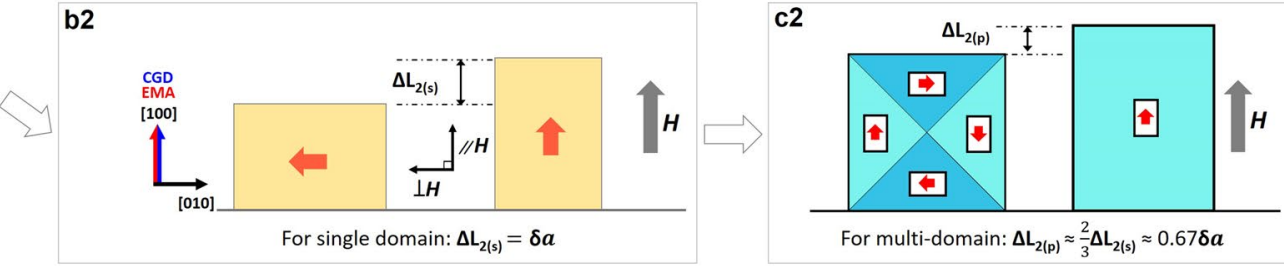

Figure 1. The schematic illustrations of the magnetostriction from two cases of $90^{\circ}$-domain switching of FeGa: the crystal growth direction (CGD) is same with/different from the easy magnetization axis (EMA). (a) The normal elongation of the crystal along EMA below Curie temperature; (b1) the single domain switching with the CGD deviating from the EMA (CGD along [110] and EMA along [100]); (b2) the single domain switching with the CGD parallel to the EMA (CGD along [100] and EMA along [100]); (c1) the multi-domain case for (b1); (c2) the multi-domain case for (b2). The red arrows represent the direction of EMA.

\section{Results}

The proposed magnetostrictive model based on $90^{\circ}$-domain switching. The $\mathrm{Fe}_{1-x} \mathrm{Ga}_{x}(x<0.2)$ possesses a body-centered cubic (BCC) A2 structure, with $\mathrm{Fe}$ and $\mathrm{Ga}$ atoms distributed disorderedly ${ }^{5}$; the EMA is along $\langle 100\rangle^{20}$ and the CGD is along $\langle 110\rangle^{21}$. Herein, we have considered the EMA along [100] and CGD along [110] to conveniently illustrate and describe the magnetostrictive model, which does not influence the proposed methodology. In the case of CGD deviating from EMA, the corresponding magnetostrictive process is illustrated in Fig. 1a,b1,cl. Figure 1a presents the normal elongation of the crystal along EMA below Curie temperature, i.e., $\delta a$, where $a$ refers to the lattice constant and $\delta$ represents the elongation coefficient of the lattice distortion. The $90^{\circ}$-domain switching ends with the external magnetic field $(\boldsymbol{H})$ parallel to the CGD [110]. If a single domain state is considered (Fig. 1b1), the magnetostriction along $\boldsymbol{H}$ is calculated to be $\sim \frac{\sqrt{2}}{2}$. $\delta a$. The calculations of the magnetostriction $\left(\Delta \mathrm{L}_{1(\mathrm{~s})}\right.$ in Fig. 1b1) are as below:

$$
\begin{aligned}
\Delta L_{1(S)} & =L_{D}-2 \cdot h \\
& =[a \cdot \cos \theta+(1+\delta) \cdot a \cdot \sin \theta]-2 \cdot a \cdot \sin \theta \\
& =a \cdot[\cos \theta+(1+\delta) \cdot \sin \theta-2 \cdot \sin \theta] \\
& =a \cdot[\delta \cdot \sin \theta+(\cos \theta-\sin \theta)]
\end{aligned}
$$

The experimentally measured magnetostriction $(10-100 \mathrm{ppm})$ demonstrates that $\delta$ is of the order of magnitude of $10^{-4}$. Therefore, the angle $\theta$ is approximately equal to $45^{\circ}$. Consequently, the magnetostriction $\Delta \mathrm{L}_{1(\mathrm{~s})}$ is:

$$
\begin{aligned}
\Delta L_{1(S)} & \approx a \cdot\left[\delta \cdot \sin 45^{\circ}+\left(\cos 45^{\circ}-\sin 45^{\circ}\right)\right] \\
& =a \cdot \delta \cdot \sin 45^{\circ} \\
& =\frac{\sqrt{2}}{2} \delta a
\end{aligned}
$$

If a multi-domain state is considered (Fig. 1c1), the magnetostriction is equal to the elongation multiplied with a domain configuration factor. For simplicity, the domain configuration factor is fixed as $2 / 3$, corresponding to the homogeneous distribution of domains along six axes of the BCC structure. Hence, the magnetostriction for the multi-domain state is calculated to be $\sim 0.47 \delta \mathrm{a}$.

In another case, if the CGD is parallel to the EMA along [100], the shape distortion of the $90^{\circ}$-domain switching completely contributes to the measured magnetostriction. This is illustrated in Fig. 1a,b2,c2. The $90^{\circ}$-domain switching ends with the external magnetic field $\boldsymbol{H}$ parallel to the EMA [100]. For the $90^{\circ}$ switching of a single domain, the value of magnetostriction is equal to $\delta a$ (Fig. 1b2). If a multi-domain state is considered (Fig. 1c2), the calculated magnetostriction is $\sim 0.67 \delta a$, which is obtained by multiplying the multi-domain factor $(2 / 3)$. In brief, if the CGD [110] can be tuned to the EMA [100], a large improvement in magnetostriction is expected.

However, the experimental realization of such domain switching process remains a challenge. Given the relationship between surface formation energy and the growth surface (growth plane) that correlates to the crystal growth direction, the preferred growth direction can be determined from the plane with the lowest surface formation energy ${ }^{22,23}$. Therefore, if elemental doping can tune the plane with the lowest surface formation energy of FeGa alloy, from (110) to (100), it is expected to improve the magnetostriction. 


\begin{tabular}{|c|c|c|c|c|c|c|c|c|}
\hline & Surface & No & 1 & 2 & 3 & 4 & Average $E$ & $\bar{E}_{s}\left(\mathrm{eV} / \AA^{2}\right)$ \\
\hline \multirow{4}{*}{$\mathrm{Fe}-\mathrm{Ga}$} & \multirow{2}{*}{$\left(\begin{array}{lll}1 & 0 & 0\end{array}\right)$} & $\mathrm{E}(\mathrm{eV})$ & -379.5764 & -382.6708 & -384.1361 & -380.7047 & -381.7720 & \multirow{2}{*}{0.1320} \\
\hline & & $\mathrm{S}\left(\AA^{2}\right)$ & 68.1251 & 69.2346 & 69.2287 & 68.1038 & 68.6730 & \\
\hline & \multirow{2}{*}{$\left(\begin{array}{lll}1 & 1 & 0\end{array}\right)$} & $\mathrm{E}(\mathrm{eV})$ & -773.8652 & -773.6429 & -773.8266 & & -773.7782 & \multirow{2}{*}{0.1274} \\
\hline & & $\mathrm{S}\left(\AA^{2}\right)$ & 102.2652 & 102.3563 & 101.9178 & & 102.1798 & \\
\hline \multirow{4}{*}{$\mathrm{Fe}-\mathrm{Ga}-\mathrm{Pt}$} & \multirow{2}{*}{$\left(\begin{array}{lll}1 & 0 & 0\end{array}\right)$} & $\mathrm{E}(\mathrm{eV})$ & -382.9563 & -384.4188 & -386.0369 & -382.5636 & -383.9939 & \multirow{2}{*}{0.1259} \\
\hline & & $\mathrm{S}\left(\AA^{2}\right)$ & 8.2348 & 8.3234 & 8.3195 & 8.2650 & 8.2857 & \\
\hline & \multirow{2}{*}{$\left(\begin{array}{lll}1 & 1 & 0\end{array}\right)$} & $\mathrm{E}(\mathrm{eV})$ & -775.7081 & -775.3140 & -775.8527 & & -775.6249 & \multirow{2}{*}{0.1319} \\
\hline & & $\mathrm{S}\left(\AA^{2}\right)$ & 102.4392 & 102.7928 & 102.3055 & & 102.5125 & \\
\hline
\end{tabular}

Table 1. Total energy of different slab structures before and after Pt doping.

The DFT calculations. In the following, the DFT calculations are carried out to demonstrate that Pt doping into FeGa crystal tunes the plane with the lowest surface formation energy from (110) to (100). Considering the large spin-orbit coupling of the $5 d$ element Pt and the potentially strong interactions between Pt and $\mathrm{Fe}$, which possesses strong spin polarization, $\mathrm{Pt}$ is chosen as the doping element to tune the CGD of FeGa crystal ${ }^{24}$. Since $\mathrm{Fe}_{0.83} \mathrm{Ga}_{0.17}$ have exhibited relatively large magnetostriction within a wide temperature range and can endure large tensile stresses, our samples are fixed as $\left(\mathrm{Fe}_{0.83} \mathrm{Ga}_{0.17}\right)_{100-x} \mathrm{Pt}_{x}$, where $\mathrm{Pt}$ substitutes the $\mathrm{Fe}$ and $\mathrm{Ga}$ sites within the A2 matrix ${ }^{3}$. Owing to the random disorder A2 structures of $\mathrm{Fe}_{0.83} \mathrm{Ga}_{0.17}{ }^{25}$, special quasirandom structure (SQS) approximation was used to construct a $3 \times 3 \times 3$ BCC supercell, which has the stoichiometry of $\mathrm{Fe}_{45} \mathrm{Ga}_{9}$, whereas the nominal composition is $\mathrm{Fe}_{0.83} \mathrm{Ga}_{0.17}{ }^{26}$. The bulk structure of $\mathrm{Fe}_{45} \mathrm{Ga}_{9}$ was completely relaxed. Based on the relaxed bulk structure, six-layered atomic slabs of FeGa, perpendicular to (100) and (110) surfaces, were built with different surface configurations. Only one Pt atom was doped into each surface of FeGa slabs to examine the influence of Pt. The total energy and surface area of the above-mentioned structures are summarized in Table 1 . The surface formation energy can be defined as $E_{s}=\frac{E_{s l a b}-E_{b u l k}}{2 S}$, where $E_{s l a b}$ and $E_{b u l k}$ refer to the total energy of slab and bulk, respectively, and $S$ represents the surface area. Considering multiple surface configurations, $E_{S}$ is determined by an average value of all slabs. As illustrated in Fig. 2, only one slab is depicted as a representative. The surface energy of (100) and (110) in FeGa slab is $\bar{E}_{S(100)}=132 \mathrm{meV} / \AA^{2}$ and $\bar{E}_{S(110)}=127.4 \mathrm{meV} / \AA^{2}$, respectively (Fig. 2a,b). The difference between the surface energy of (100) and (110) is $\Delta \bar{E}_{S}=\bar{E}_{S(100)}-\bar{E}_{S(110)}=4.6 \mathrm{meV} / \AA^{2}$, which implies that (110) is more stable than (100) and easily forms during solidification. The replacement of $\mathrm{Fe}$ or $\mathrm{Ga}$ by $\mathrm{Pt}$ on the surface of the above slabs hardly changes the atomic structure, as shown in Fig. 2c,d. However, the surface formation energy of (100) and (110) is changed to $\bar{E}_{S-P t(100)}=125.9 \mathrm{meV} / \AA^{2}$ and $\bar{E}_{S-P t(110)}=131.9 \mathrm{meV} / \AA^{2}$, respectively. Hence, the difference in surface formation energies becomes $\Delta \bar{E}_{S-P t}=\bar{E}_{S-P t(100)}-\bar{E}_{S-P t(110)}=-6.0 \mathrm{meV} / \AA^{2}$. Apparently, the surface formation energy relationship is reversed, which indicates that (100) is more stable than (110) and becomes a preferred growth plane after Pt doping. The DFT calculations indicate that doping of Pt can tune the CGD of FeGa from [110] to [100] (parallel to the EMA), which is expected to improve the magnetostriction, as depicted in Fig. 1.

X-ray diffraction analysis. For verification, we prepared both as-cast and directionally solidified (DS) Ptdoped FeGa crystals. The X-ray diffraction (XRD) patterns of the selected compositions are shown in Fig. 3a. The as-cast and the DS-treated samples exhibit only characteristic (110), (200) and (211) peaks within the 2theta range from $30^{\circ}$ to $90^{\circ}$, demonstrating that pure body-centered cubic (BCC) A2 structure is retained after Pt doping ${ }^{15,27}$. Figure $3 \mathrm{~b}$ shows the Pt content dependence of lattice constant. With the increase of Pt content, the lattice constants of both as-cast and DS-treated FeGa crystals increase to the maximum at $x=0.8$ and then decrease at $x=1.0$. Figure $3 c$ presents the Pt content dependence of the intensity ratio $\left(\mathrm{I}_{200} / \mathrm{I}_{110}\right)$ between $(200)$ and (110) peaks. The maximum of $\mathrm{I}_{200} / \mathrm{I}_{110}$ appears at $x=0.8$ for both as-cast and DS-treated crystals, $43.1 \%$ and $380.3 \%$, respectively. When Pt content exceeds $0.8, \mathrm{I}_{200} / \mathrm{I}_{110}$ decreases to $26.5 \%$ and $34.9 \%$ at $x=1.0$, for as-cast and DS-treated samples, respectively. The decrease of the lattice constant and the orientation preference along [100] when Pt content exceeds 0.8, may be caused by the solubility limit of Pt in FeGa crystal. It is clear that before and after Pt doping, the samples maintain polycrystalline state, and the results of the intensity ratio of $\mathrm{I}_{200} / \mathrm{I}_{110}$ demonstrate the favorable growth direction along [100] with Pt doping up to 0.8 at.\%, which is in good agreement with DFT calculations.

Magnetostriction and magneto-crystalline anisotropic constant $\mathrm{K}_{1}$. Figure $4 \mathrm{a}$ presents the $\mathrm{Pt}$ content dependent magnetostriction curves at room temperature. The un-doped FeGa sample $(x=0)$ shows the magnetostriction of $39 \mathrm{ppm}$, which is consistent with the previously reported values of $20-60 \mathrm{ppm}$ and theoretical prediction ${ }^{28}$. The composition $x=0.8$ shows the highest magnetostriction of $103 \mathrm{ppm}$ for as-cast sample and $188 \mathrm{ppm}$ for DS-treated sample. It should be noted that as for the trace-amount element doped FeGa polycrystalline alloys without treatment of magnetic annealing and prestress during measurement, the reported maximum value of magnetostriction (even DS-treated FeGa alloys) is $160 \mathrm{ppm}^{29-34}$. Meanwhile, the Pt doping causes a higher magnetostriction without increasing the saturation field, which can be quantified as $\mathrm{d} \lambda / \mathrm{d} \boldsymbol{H}$, as shown in Fig. 4b. For as-cast and DS-treated samples, the largest $\mathrm{d} \lambda / \mathrm{d} \boldsymbol{H}$ appears at $x=0.8$ and reaches the maximum value of $0.11 \mathrm{ppm} / \mathrm{Oe}$ and $0.17 \mathrm{ppm} / \mathrm{Oe}$, respectively. This feature is also desirable in practical applications. The higher 


\section{$\mathrm{Fe} \odot \mathrm{Ga} \odot \mathrm{Pt}$}

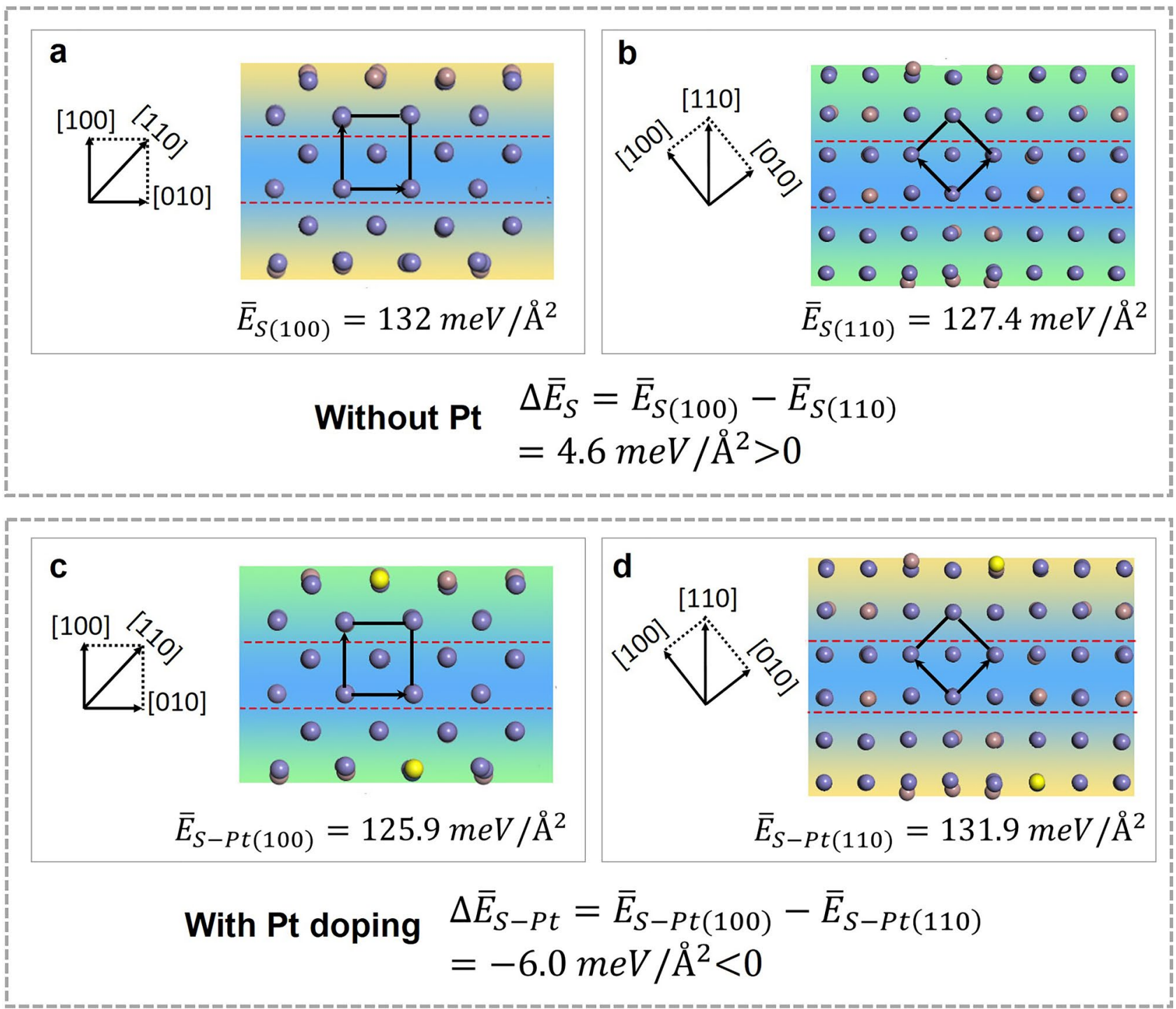

Figure 2. The surface formation energies determined by DFT calculations: side view of atomic structures for (a) FeGa alloy slab along [100], (b) FeGa alloy slab along [110], (c) FeGa alloy slab along [100] with Pt doping on its surface, (d) FeGa alloy slab along [110] with Pt doping on its surface. Orange regions correspond to the higher energy surfaces, whereas the green regions represent the lower energy surfaces. The blue regions refer to the interior of crystals.

the value of $\mathrm{d} \lambda / \mathrm{d} \boldsymbol{H}$ is, the lower field is needed to trigger large magnetostriction. In practical applications, the magnetic materials with higher value of $\mathrm{d} \boldsymbol{\lambda} / \mathrm{d} \boldsymbol{H}$ can help realize the miniaturization of devices.

Because the samples are polycrystalline, the magneto-crystalline anisotropic constant $\mathrm{K}_{1}$ cannot be measured directly. Using the methods proposed by Vazquez et al. and Andreev et al. ${ }^{35,36}$, the magneto-crystalline anisotropic constant $K_{1}$ is calculated from the magnetization as a function of magnetic field (Fig. $4 \mathrm{c}$, details can be referred to the supplementary materials). For our samples, the calculated $\mathrm{K}_{1}$ ranges $3.7-4.0 \times 10^{5} \mathrm{erg} / \mathrm{cm}^{3}$, while Rafique. S et al. have reported that the $\mathrm{K}_{1}$ of $\mathrm{FeGa}$ alloy ranges $3-7 \times 10^{4} \mathrm{~J} / \mathrm{m}^{337}$. Considering the relation that $1 \mathrm{erg} / \mathrm{cm}^{3}=10^{-1} \mathrm{~J} / \mathrm{m}^{3}$, the values of calculated $\mathrm{K}_{1}$ for our FeGa samples are in good agreement with the previous reports. From the inset of Fig. $4 \mathrm{c}$, it can be seen that compared with $x=0.8$, the sample of $x=0$ is harder to be magnetized, reflected from both the saturated magnetization and the slope of the curve. This result agrees well with Fig. 1c1,c2. The FeGa- $x$ Pt alloy of $x=0.8$ exhibits the highest magneto-crystalline anisotropic constant $\mathrm{K}_{1}$ of $3.97{ }^{\star} 10^{5} \mathrm{erg} / \mathrm{cm}^{3}$ and $4.00 * 10^{5} \mathrm{erg} / \mathrm{cm}^{3}$ for as-cast and DS-treated samples, respectively. The trend of $\mathrm{K}_{1}$ variation with $\mathrm{Pt}$ content is also consistent with the intensity ratio $\mathrm{I}_{200} / \mathrm{I}_{110}$. The increase of $\mathrm{K}_{1}$ with the increase of Pt content, up to $x=0.8$, demonstrates the increase of magneto-crystalline anisotropy and, consequently, the increase of $\delta$ (Fig. 1), which further improves the magnetostriction. 

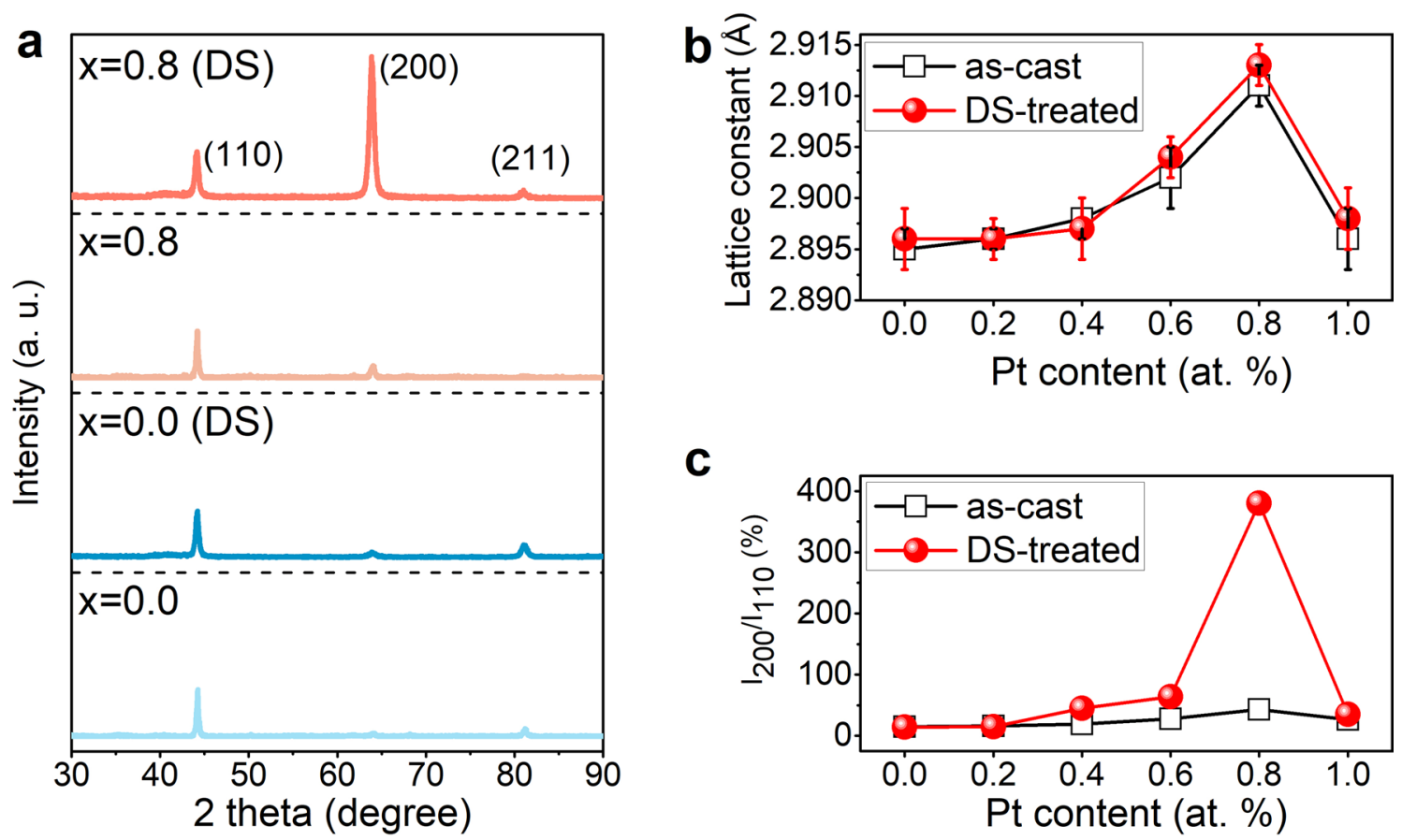

Figure 3. XRD patterns of $\left(\mathrm{Fe}_{0.83} \mathrm{Ga}_{0.17}\right)_{100-x} \mathrm{Pt}_{x}$ alloys: (a) $x=0$ and 0.8 for as-cast and DS-treated samples; (b) the Pt dependence of the intensity ratio between (200) peak and (110) peak for as-cast and DS-treated samples; (c) the Pt dependence of the lattice constant for as-cast and DS-treated samples.
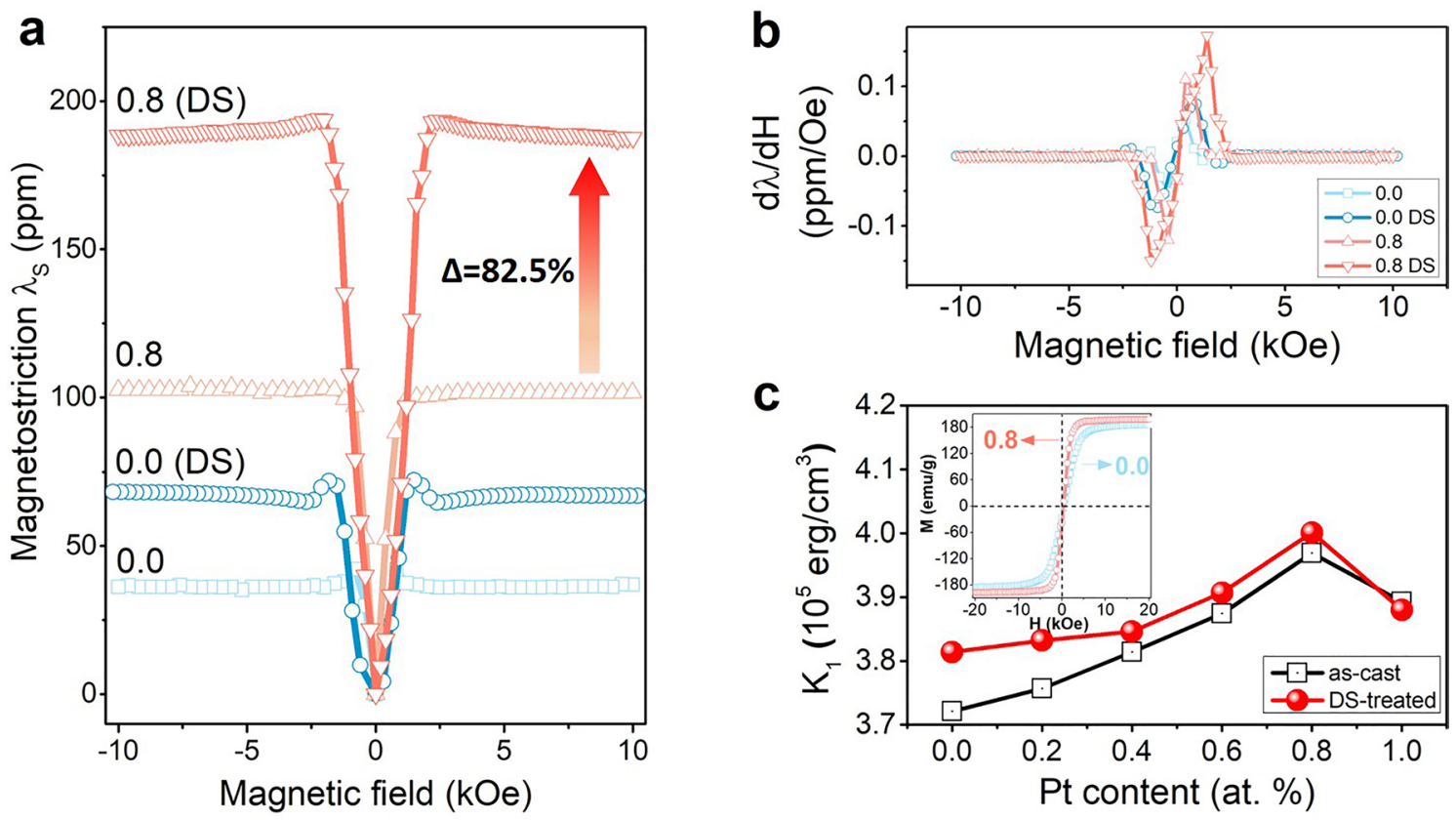

Figure 4. The magnetostriction curves and magneto-crystalline anisotropy constant $\mathrm{K}_{1}$ of $\left(\mathrm{Fe}_{0.83} \mathrm{Ga}_{0.17}\right)_{100-x} \mathrm{Pt}_{x}$ alloys: (a) $x=0$ and 0.8 for as-cast and DS-treated samples; (b) first order derivatives of magnetostriction over magnetic field ( $x=0$ and 0.8 for as-cast and DS-treated samples); (c) the calculated magneto-crystalline anisotropic constant $\mathrm{K}_{1}$ with the inset showing the $\boldsymbol{M}-\boldsymbol{H}$ hysteresis loops ( $x=0$ and 0.8 ).

Discussion. It should be noticed that, for the same composition, e.g., $x=0$, the DS-treated sample and the as-cast sample exhibit different magnetostrictions while there are no obvious differences in $\mathrm{I}_{200} / \mathrm{I}_{110}$ (Figs. 3c, 4a). Considering that DS is one type of heat treatment process, the differences between $x=0$ (as-cast) and $x=0$ (DStreated) are probably due to the nanoheterogeneities (m-D03 phase nano-inclusions or precipitates) that have been studied extensively ${ }^{11-14,38}$. 
Last but not least, let us return to the previously reported methods to improve the magnetostriction of FeGa alloys. The XRD patterns of the FeGa alloys prepared by magnetic annealing ${ }^{15}$, melt-spinning 9,39 , and single crystal growth ${ }^{27}$ all indicate the positive correlation between the magnetostriction and the preferred growth direction along [100]. This validates our proposed model and indicates the presence of a common physical mechanism behind these different approaches. Compared with previous work, the fundamental scientific objective of the present work is to tune the intrinsic factor to enhance the magnetostriction, without considering complex treatments and measurement auxiliary conditions. Besides, with the assistance of DFT calculations, the design and fabrication of high-performance FeGa alloys will be more efficient.

In conclusion, based on a crystallographic geometric model, we propose an approach to improving the magnetostriction of FeGa alloys by aligning the crystal growth direction with the easy magnetization axis, and the DFT calculations indicate that the doping of Pt can facilitate such process through tuning the crystal surface formation energy. Furthermore, the proposed model is experimentally verified: accompanying with the increasing preference of the crystal growth along [100] direction, the magnetostriction of FeGa with Pt doping is greatly improved from $39 \mathrm{ppm}(x=0)$ to $103 \mathrm{ppm}(x=0.8)$ and $188 \mathrm{ppm}(x=0.8$, DS). The present study provides an effective approach to explore and design high-performance magnetostrictive materials.

\section{Methods}

Sample preparation and characterization. The $\left(\mathrm{Fe}_{0.83} \mathrm{Ga}_{0.17}\right)_{100-x} \mathrm{Pt}_{x}$ polycrystalline samples (x represents the atomic percentages; $x=0,0.2,0.4,0.6,0.8$ and 1.0$)$ were prepared by using high purity metals of $\mathrm{Fe}$ (99.95\%), Ga (99.99\%) and Pt (99.99\%) by arc-melting techniques under argon atmosphere. Considering the lower melting point of $\mathrm{Ga}$, excessive $1 \mathrm{~mol} \%$ of $\mathrm{Ga}$ was added to compensate the losses during the melting process. To ensure compositional homogeneity, each sample $(\sim 7 \mathrm{~g})$ was melted four times and the weight loss of the ingots was less than $1 \%$. The as-cast ingots were sectioned into slices with a thickness of $1 \mathrm{~mm}$ by wirecutting and sealed into a quartz tube, filled with argon gas, followed by a heat treatment at $1000{ }^{\circ} \mathrm{C}$ for $3 \mathrm{~h}$, and then quenched into water. The directionally solidified samples were prepared at $1680^{\circ} \mathrm{C}$ with the pulling rate of $5 \mu \mathrm{m} / \mathrm{s}$. The X-ray diffraction (XRD) patterns were measured by using a Bruker D8 ADVANCE Diffractometer $(\mathrm{Cu}-\mathrm{K} \alpha, \lambda=1.5406 \AA)$ and the lattice constants of FeGa-Pt alloys were calculated by using Nielsen extrapolation method.

Property measurements. The magnetic characterization was carried out on the superconducting quantum interference device-vibrating sample magnetometer (MPMS-SQUID VSM-094). The magnetostriction was tested with a standard strain gauge at room temperature.

Computational methods. The Vienna Ab-initio Simulation Package (VASP) was used to conduct DFT calculations with the projector augmented wave $\operatorname{method}^{40}$. The spin-polarized generalized gradient approximation (GGA) with the Perdew-Burke-Ernzerhof (PBE) functional was adopted ${ }^{41}$. The kinetic energy cutoff for wavefunction expansion was set to $400 \mathrm{eV}$. Monkhorst-Pack k-point grid of $3 \times 3 \times 3$ and $3 \times 3 \times 1$ was used for bulk and slab supercell, respectively. Atomic positions and lattice constants were completely relaxed in bulk. For the slab models, two surface atomic layers were completely relaxed, but two middle atomic layers were fixed in lattice constants of the relaxed bulk model to simulate the surface influence. Both relaxations were terminated once the difference of energy and force was less than $10^{-5} \mathrm{eV}$ and $0.01 \mathrm{eV} / \AA$, respectively. The periodic boundary conditions were applied in calculations, while the vacuum space was added along the $\mathrm{z}$-axis for slab model at least $20 \AA$ to safely avoid artificial interaction between the periodic images.

\section{Data availability}

The data that support the findings of this study are available from the corresponding authors on request.

Received: 26 June 2020; Accepted: 16 October 2020

Published online: 18 November 2020

\section{References}

1. Clark, A. E. Ferromagnetic Materials-A Handbook on the Properties of Magnetically Ordered Substances, Vol. 1531-589 (NorthHolland Publishing Company, Amsterdam, 1980).

2. Engdahl, G. Handbook of Giant Magnetostrictive Materials (Academic Press, San Diego, CA, 2000).

3. Clark, A. E., Restorff, J. B., Wun-Fogle, M., Lograsso, T. A. \& Schlagel, D. L. Magnetostrictive properties of body-centered cubic $\mathrm{Fe}-\mathrm{Ga}$ and Fe-Ga-Al alloys. IEEE Trans. Magn. 36, 3238-3240. https://doi.org/10.1109/20.908752 (2000).

4. Cullen, J. R., Clark, A. E., Wun-Fogle, M., Restorff, J. B. \& Lograsso, T. A. Magnetoelasticity of Fe-Ga and Fe-Al alloys. J. Magn. Magn. Mater. 226-230, 948-949. https://doi.org/10.1016/S0304-8853(00)00612-0 (2001).

5. Clark, A. E., Wun-Fogle, M., Restorff, J. B. \& Lograsso, T. A. Magnetostrictive properties of galfenol alloys under compressive stress. Mater. Trans. 43, 881-886. https://doi.org/10.2320/matertrans.43.881 (2002).

6. Kellogg, R. A., Flatau, A. B., Clark, A. E., Wun-Fogle, M. \& Lograsso, T. A. Temperature and stress dependencies of the magnetic and magnetostrictive properties of $\mathrm{Fe}_{0.81} \mathrm{Ga}_{0.19}$. J. Appl. Phys. 91, 7821-7823. https://doi.org/10.1063/1.1452216 (2002).

7. Clark, A. E. et al. Extraordinary magnetoelasticity and lattice softening in bcc Fe-Ga alloys. J. Appl. Phys. 93, 8621-8623. https:// doi.org/10.1063/1.1540130(2003).

8. Yao, Z. et al. Influences of rare earth element Ce-doping and melt-spinning on microstructure and magnetostriction of $\mathrm{Fe}_{83} \mathrm{Ga}_{17}$ alloy. J. Alloys Compd. 637, 431-435. https://doi.org/10.1016/j.jallcom.2015.03.009 (2015).

9. Jin, T., Wu, W. \& Jiang, C. Improved magnetostriction of Dy-doped $\mathrm{Fe}_{83} \mathrm{Ga}_{17}$ melt-spun ribbons. Scr. Mater. 74, 100-103. https:// doi.org/10.1016/j.scriptamat.2013.11.010 (2014).

10. Wu, W., Liu, J., Jiang, C. \& Xu, H. Giant magnetostriction in Tb-doped $\mathrm{Fe}_{83} \mathrm{Ga}_{17}$ melt-spun ribbons. Appl. Phys. Lett. 103, 262403. https://doi.org/10.1063/1.4851216 (2013). 
11. He, Y. et al. Giant heterogeneous magnetostriction in Fe-Ga alloys: Effect of trace element doping. Acta Mater. 109, 177-186. https ://doi.org/10.1016/j.actamat.2016.02.056 (2016).

12. Meng, C., Wu, Y. \& Jiang, C. Design of high ductility FeGa magnetostrictive alloys: Tb doping and directional solidification. Mater. Des. 130, 183-189. https://doi.org/10.1016/j.matdes.2017.05.053 (2017).

13. Ke, Y., Jiang, C., Tao, J. \& Duan, H. Local inhomogeneous structural origin of giant magnetostriction in Fe-Ga alloys. J. Alloys Compd. 725, 14-22. https://doi.org/10.1016/j.jallcom.2017.05.180 (2017).

14. He, Y. et al. Interaction of trace rare-earth dopants and nanoheterogeneities induces giant magnetostriction in $\mathrm{Fe}-\mathrm{Ga}$ alloys. Adv. Funct. Mater. 28, 1800858. https://doi.org/10.1002/adfm.201800858 (2018).

15. Wen, S. et al. Magnetostriction enhancement by high magnetic field annealing in cast $\mathrm{Fe}_{81} \mathrm{Ga}_{19}$ alloy. J. Magn. Magn. Mater. 442, 128-135. https://doi.org/10.1016/j.jmmm.2017.06.095 (2017).

16. Srisukhumbowornchai, N. \& Guruswamy, S. Large magnetostriction in directionally solidified FeGa and FeGaAl alloys. J. Appl. Phys. 90, 5680-5688. https://doi.org/10.1063/1.1412840 (2001).

17. Atulasima, J. Energy-based quasi-static modeling of the actuation and sensing behavior of single-crystal iron-gallium alloys. J. Appl. Phys. 103, 014901. https://doi.org/10.1063/1.2826946 (2008).

18. Clark, A. E. \& Wun-Fogle, M. In SPIE's 9th Annual International Symposium on Smart Structures and Materials 421.

19. Lacheisserie, E. D. T. D. Magnetostriction: Theory and Applications of Magnetoelasticity (CRC Press, Boca Raton, 1993).

20. Kawamiya, N., Adachi, K. \& Nakamura, Y. Magnetic properties and Mössabauer investigations of Fe-Ga alloys. J. Phys. Soc. Jpn. 33, 1318-1327. https://doi.org/10.1143/JPSJ.33.1318 (1972).

21. Fitchorov, T. I. et al. Thermally driven large magnetoresistance and magnetostriction in multifunctional magnetic FeGa-Tb alloys. Acta Mater. 73, 19-26. https://doi.org/10.1016/j.actamat.2014.03.053 (2014).

22. Rohl, A. L. \& Gay, D. H. Calculating the effects of surface relaxation on morphology. J. Cryst. Growth 166, 84-90. https://doi. org/10.1016/0022-0248(96)00118-2 (1996).

23. Zhu, W. \& Wu, P. Surface energetics of hydroxyapatite: A DFT study. Chem. Phys. Lett. 396, 38-42. https://doi.org/10.1016/j.cplet t.2004.08.006 (2004).

24. Zhang, Y. N. \& Wu, R. Q. Large magnetostriction in Fe-based alloys predicted by density functional theory. Phys. Rev. B 82, 224415. https://doi.org/10.1103/PhysRevB.82.224415 (2010).

25. Cullen, J., Zhao, P. \& Wuttig, M. Anisotropy of crystalline ferromagnets with defects. J. Appl. Phys. 101, 123922. https://doi. org/10.1063/1.2749471 (2007).

26. Zunger, A., Wei, S. H., Ferreira, L. G. \& Bernard, J. E. Special quasirandom structures. Phys. Rev. Lett. 65, 353-356. https://doi. org/10.1103/PhysRevLett.65.353 (1990).

27. Meng, C. \& Jiang, C. Magnetostriction of a $\mathrm{Fe}_{83} \mathrm{Ga}_{17}$ single crystal slightly doped with Tb. Scr. Mater. 114, 9-12. https://doi. org/10.1016/j.scriptamat.2015.11.022 (2016).

28. Wang, Z.-B., Liu, J.-H. \& Jiang, C.-B. Magnetostriction of $\mathrm{Fe}_{81} \mathrm{Ga}_{19}$ oriented crystals. Chin. Phys. B 19, 117504 (2010).

29. Xu, S. F. et al. Magnetostriction and electrical resistivity of $\mathrm{Mn}$ doped $\mathrm{Fe}_{81} \mathrm{Ga}_{19}$ alloys. J. Phys. D Appl. Phys. 41, 015002 (2008)

30. Ji-heng, L., Xue-xu, G., Jie, Z., Jie, L. \& Mao-cai, Z. Effect of boron and chromium on magnetostriction and mechanical properties of polycrystalline $\mathrm{Fe}_{83} \mathrm{Ga}_{17}$ alloy. J. Univ. Sci. Technol. Beijing 31, 5 (2009).

31. Zhou, Y. et al. The magnetostriction of Fe- $(18-\mathrm{x})$ at $\% \mathrm{Ga}-\mathrm{xat} \% \mathrm{Al}(3 \leq \mathrm{x} \leq 13.5)$ alloys. J. Magn. Magn. Mater. 322, 2104-2107. https ://doi.org/10.1016/j.jmmm.2010.01.041 (2010).

32. Bormio-Nunes, C. et al. Improved magnetostriction of $\mathrm{Fe}_{72} \mathrm{Ga}_{28}$ boron doped alloys. J. Appl. Phys. 109, 07A934. https://doi. org/10.1063/1.3561782 (2011).

33. Jiang, L. et al. Giant enhancement in the magnetostrictive effect of FeGa alloys doped with low levels of terbium. Appl. Phys. Lett. 102, 222409. https://doi.org/10.1063/1.4809829 (2013).

34. Taheri, P. et al. Structure, magnetism, and magnetostrictive properties of mechanically alloyed $\mathrm{Fe}_{81} \mathrm{Ga}_{19}$. J. Alloys Compd. 661, 306-311. https://doi.org/10.1016/j.jallcom.2015.11.037 (2016).

35. Andreev, S. V. et al. Law of approach to saturation in highly anisotropic ferromagnets application to NdFeB melt-spun ribbons. J. Alloys Compd. 260, 196-200. https://doi.org/10.1016/S0925-8388(97)00127-8 (1997).

36. Vazquez, M., Fernengel, W. \& Kronmüller, H. Approach to magnetic saturation in rapidly quenched amorphous alloys. Phys. Status Solidi (a) 115, 547-553. https://doi.org/10.1002/pssa.2211150223 (1989).

37. Rafique, S., Cullen, J. R., Wuttig, M. \& Cui, J. Magnetic anisotropy of FeGa alloys. J. Appl. Phys. 95, 6939-6941. https://doi. org/10.1063/1.1676054 (2004).

38. Wu, Y. et al. Multiscale influence of trace $\mathrm{Tb}$ addition on the magnetostriction and ductility of $\langle 100\rangle$ oriented directionally solidified Fe-Ga crystals. Phys. Rev. Mater. 3, 033401. https://doi.org/10.1103/PhysRevMaterials.3.033401 (2019).

39. Wu, W., Liu, J. \& Jiang, C. Tb solid solution and enhanced magnetostriction in $\mathrm{Fe}_{83} \mathrm{Ga}_{17}$ alloys. J. Alloys Compd. 622, 379-383. https://doi.org/10.1016/j.jallcom.2014.09.151 (2015).

40. Kresse, G. \& Furthmüller, J. Efficient iterative schemes for ab initio total-energy calculations using a plane-wave basis set. Phys. Rev. B 54, 11169-11186. https://doi.org/10.1103/PhysRevB.54.11169 (1996).

41. Perdew, J. P., Burke, K. \& Ernzerhof, M. Generalized gradient approximation made simple. Phys. Rev. Lett. 77, 3865-3868. https ://doi.org/10.1103/PhysRevLett.77.3865 (1996)

\section{Acknowledgements}

The authors are grateful to Professor J. M. D. Coey for helpful discussions. This work was financially supported by the National Natural Science Foundation of China (Grants Nos. 91963111, 51601140, 51701149, 51871174 and 51728203), Key Scientific and Technological Innovation Team of Shaanxi Province (2020TD-001), Innovation Capability Support Program of Shaanxi (Nos. 2018PT-28, 2017KTPT-04), the Fundamental Research Funds for the Central Universities (China) and the World-Class Universities (Disciplines), the Characteristic Development Guidance Funds for the Central Universities (China).

\section{Author contributions}

C.Z., T.M. and S.Y. conceived the model and designed the experiments. Y.L., and Z.D., S.R., R.Z. and F.T. synthesized the samples, characterized the samples by using X-ray diffraction and performed the magnetostriction measurement. Y.W., K.C., Y.Z. and H.Z. performed the density functional theory calculations and data analysis. C.Z., Y.L., J.D., and S.Y. wrote the manuscript. All the authors commented on the manuscript.

\section{Competing interests}

The authors declare no competing interests. 


\section{Additional information}

Supplementary information is available for this paper at https://doi.org/10.1038/s41598-020-77058-2.

Correspondence and requests for materials should be addressed to J.D. or S.Y.

Reprints and permissions information is available at www.nature.com/reprints.

Publisher's note Springer Nature remains neutral with regard to jurisdictional claims in published maps and institutional affiliations.

Open Access This article is licensed under a Creative Commons Attribution 4.0 International License, which permits use, sharing, adaptation, distribution and reproduction in any medium or format, as long as you give appropriate credit to the original author(s) and the source, provide a link to the Creative Commons licence, and indicate if changes were made. The images or other third party material in this article are included in the article's Creative Commons licence, unless indicated otherwise in a credit line to the material. If material is not included in the article's Creative Commons licence and your intended use is not permitted by statutory regulation or exceeds the permitted use, you will need to obtain permission directly from the copyright holder. To view a copy of this licence, visit http://creativecommons.org/licenses/by/4.0/.

(c) The Author(s) 2020 